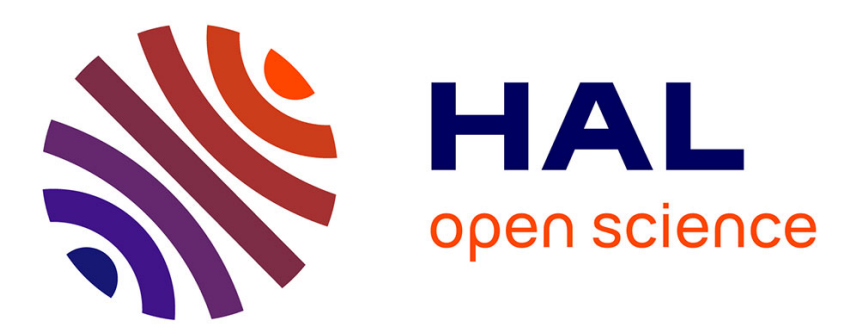

\title{
Robust reduced order unbiased filtering for uncertain systems
}

Michel Zasadzinski, Harouna Souley Ali, Mohamed Darouach

\section{To cite this version:}

Michel Zasadzinski, Harouna Souley Ali, Mohamed Darouach. Robust reduced order unbiased filtering for uncertain systems. International Journal of Control, 2006, 79 (2), pp.93-106. hal-00090252

\section{HAL Id: hal-00090252 \\ https://hal.science/hal-00090252}

Submitted on 29 Aug 2006

HAL is a multi-disciplinary open access archive for the deposit and dissemination of scientific research documents, whether they are published or not. The documents may come from teaching and research institutions in France or abroad, or from public or private research centers.
L'archive ouverte pluridisciplinaire HAL, est destinée au dépôt et à la diffusion de documents scientifiques de niveau recherche, publiés ou non, émanant des établissements d'enseignement et de recherche français ou étrangers, des laboratoires publics ou privés. 


\title{
Robust reduced order unbiased filtering for uncertain systems
}

\author{
M. Zasadzinski, H. Souley Ali, M. Darouach
}

\begin{abstract}
This paper presents a simple solution to the robust $\mathcal{H}_{\infty}$ unbiased functional reduced order filtering problem in the case of uncertain systems described by Integral Quadratic Constraint (IQC). The existence condition of the unbiased filter is expressed through a rank relation. Then the filter is designed via Linear Matrix Inequalities coupled with an equality constraint. The results obtained in this paper are applicable to unstable systems. The approach is illustrated by a numerical example.
\end{abstract}

Keywords : Robust filtering, Reduced order observers, IQC, Uncertainties, LMI, Unbiasedness.

\section{Introduction}

This paper concerns the synthesis of robust reduced order $\mathcal{H}_{\infty}$ filter for uncertain systems where the uncertain variables are described by Integral Quadratic Constraints (IQC). The robust filtering gets its importance from the necessity to still keep good performances even when uncertainties affect the nominal system. These uncertainties can result from system identification, model reduction, time delays, nonlinearities, ... IQC are shown to well describe these types of uncertainties in numerous papers, notably for signal processing applications Xie et al. (1998). Due to computational or real-time implementation reasons, reduced order filter should be prefered to the full order one, specially in practical applications for large scale systems Nagpal et al. (1987); Kim et al. (1992); Watson and Grigoriadis (1998).

Hence, we deal with the estimation of the functional $z(t)=L x(t)$, where $z(t) \in \mathbb{R}^{r}, r \leqslant n$ and $x(t) \in \mathbb{R}^{n}$ is the state of the system under consideration.

The functional unbiased filter is designed such that the $\mathcal{H}_{\infty}$ norm from the disturbance inputs to the estimation error is smaller than a given prescribed value. The problem of $\mathcal{H}_{\infty}$ filtering has been widely studied in the full order case Shaked (1990); Nagpal and Khargonekar (1991); Shaked and Theodor (1992) and even in the reduced order one Kim et al. (1992); Grigoriadis and Watson (1997); Watson and Grigoriadis (1998). The advantage of the $\mathcal{H}_{\infty}$ filtering is that it does not require any knowledge on the statiscal properties of the disturbances, they must only be of finite energy. Recall that this is not the case in the standard Kalman filtering which needs statistical informations about the disturbances.

When nominal systems are affected by uncertainties, robust filters must be designed to ensure the stability of the reconstruction error in spite of the presence of uncertainties (see Bolzern et al. (1996); Geromel (1999); Geromel et al. (2000); El Ghaoui andCalafiore (2001); Li and Fu (1997); Savkin and Petersen (1996); Wang and Balakrishnan (2002) for the full order robust filter and Savkin and Petersen (1997); Tuan et al. (2001) for the reduced order one). Note that all these robust filters are biased, i.e. the error dynamics contains a term depending directly on the system state in the nominal case. The advantage of the robust unbiased filtering in comparison to the biased one is that it ensures a best performance in the nominal case Shaked and de Souza (1995). The unbiasedness condition is derived from the error dynamics. Its goal is to suppress the direct effect of the system state in this dynamics. The unbiased robust filtering has been treated in Shaked and de Souza (1995) with a filter of order greater than that of the system and in Bittanti and Cuzzola (2000) with a filter of the same order as the system.

\footnotetext{
${ }^{*}$ Corresponding author : M. Zasadzinski, e-mail : mzasad@iut-longwy.uhp-nancy.fr, tel : 00333823962 22, fax : 0033 382396291 .

${ }^{\dagger}$ The authors are with CRAN UMR 7039-CNRS, IUT de Longwy, Université Henri Poincaré (Nancy I), 186 rue de Lorraine, 54400 Cosnes et Romain, FRANCE.
} 
In this paper, our goal is twofold. First, we establish existence and stability conditions for the robust functional filter of order $r$ in presence of uncertainties described by IQC. These conditions are given in such a way to avoid bilinearities between the filter parameters. As in Darouach (2000); Darouach et al. (2001), the presence of a direct feedthrough term in the filter state-space realization allows us to generalize the condition given in Watson and Grigoriadis (1998). A Linear Matrix Inequality (LMI) method is thus applied to solve the filtering problem as a static output feedback problem. As inherent to the later problem, the solution implies to solve also a non convex (equality) constraint Tuan et al. (2001). This is the main difference with the full order robust filtering. Under appropriate condition, we show that the presence of unstable modes can be taken into account since their effects on the estimation error vanish because of the unbiasedness condition (see Xie et al. (1994) for the full order case). Note that the choice of an $\mathcal{H}_{\infty}$ filtering performance is not intrinsic. In fact, the approach can be easily extended to the robust $\mathcal{L}_{2}-\mathcal{L}_{\infty}$ filtering case.

This paper is organized as follows. Section 2 gives necessary and sufficient conditions for the unbiasedness of a functional filter for both continuous-time and discrete-time uncertain systems. Then section 3 gives the solution to the robust $\mathcal{H}_{\infty}$ filtering problem whereas section 4 presents an illustrative example. Section 5 concludes the paper.

\section{Unbiasedness and stability conditions}

\subsection{Problem Statement}

Consider the following uncertain linear system

$$
\begin{aligned}
\sigma x_{1} & =A_{1} x_{1}+\sum_{i=1}^{s} H_{11 i} p_{i}+B_{1} w \\
\sigma x_{2} & =A_{21} x_{1}+A_{2} x_{2}+\sum_{i=1}^{s} H_{12 i} p_{i}+B_{2} w \\
y & =C_{1} x_{1}+C_{2} x_{2}+\sum_{i=1}^{s} H_{2 i} p_{i}+D w \\
z & =L x=L_{1} x_{1}+L_{2} x_{2}
\end{aligned}
$$

where $x(t)=\left[\begin{array}{ll}x_{1}(t)^{T} & x_{2}(t)^{T}\end{array}\right]^{T} \in \mathbb{R}^{n}$ (with $x_{1}(t) \in \mathbb{R}^{n_{1}}$ and $x_{2}(t) \in \mathbb{R}^{n_{2}}$ ) is the state vector, $y(t) \in \mathbb{R}^{p}$ is the measured output and $z(t) \in \mathbb{R}^{r}$ is the vector of variables to be estimated where $r \leqslant n$. The vectors $w(t) \in \mathbb{R}^{m}$ and $p_{i}(t) \in \mathbb{R}^{k_{i}}$ represent the disturbance and the uncertain variables respectively. We have $\bar{k}=\sum_{i=1}^{s} k_{i}$ where $k_{i}$ is the number of columns of matrices $H_{11 i}, H_{12 i}$ and $H_{2 i} . \sigma x(t)=\dot{x}(t)$ is used for the continuous-time case and $\sigma x(t)=x(t+1)$ for the discrete-time one. Assume without loss of generality that $\operatorname{rank} L=r$.

To describe the effect of uncertainties on system (1), we consider $s$ fictitious outputs $q_{i}(t)$ given by

$$
q_{i}=E_{11 i} x_{1}+E_{2 i} p+E_{3 i} w \quad i=1, \ldots, s .
$$

The following notations will be used in the sequel

$$
A=\left[\begin{array}{cc}
A_{1} & 0 \\
A_{21} & A_{2}
\end{array}\right], B=\left[\begin{array}{c}
B_{1} \\
B_{2}
\end{array}\right], C=\left[\begin{array}{ll}
C_{1} & C_{2}
\end{array}\right], p=\left[\begin{array}{c}
p_{1} \\
\vdots \\
p_{s}
\end{array}\right], q=\left[\begin{array}{c}
q_{1} \\
\vdots \\
q_{s}
\end{array}\right]
$$

and

$$
\left[\begin{array}{c}
H_{1} \\
H_{2}
\end{array}\right]=\left[\begin{array}{c}
H_{11} \\
H_{12} \\
\hline H_{2}
\end{array}\right]=\left[\begin{array}{ccc}
H_{111} & \ldots & H_{11 s} \\
H_{121} & \ldots & H_{12 s} \\
\hline H_{21} & \ldots & H_{2 s}
\end{array}\right],\left[\begin{array}{c}
E_{11}^{T} \\
E_{2}^{T} \\
E_{3}^{T}
\end{array}\right]=\left[\begin{array}{ccc}
E_{111}^{T} & \ldots & E_{11 s}^{T} \\
E_{21}^{T} & \ldots & E_{2 s}^{T} \\
E_{31}^{T} & \ldots & E_{3 s}^{T}
\end{array}\right]
$$


Then system (1) can be rewritten in the following compact form

$$
\begin{aligned}
\sigma x & =A x+H_{1} p+B w \\
y & =C x+H_{2} p+D w \\
z & =L x
\end{aligned}
$$

It is assumed that the uncertainties satisfy the following admissible IQC

$$
\int_{0}^{T}\left\|p_{i}(t)\right\|^{2} \mathrm{~d} t \leqslant \int_{0}^{T}\left\|E_{11 i} x_{1}(t)+E_{2 i} p(t)+E_{3 i} w(t)\right\|^{2} \mathrm{~d} t
$$

in the continuous-time case and

$$
\sum_{k=0}^{T}\left\|p_{i}(k)\right\|^{2} \leqslant \sum_{k=0}^{T}\left\|E_{11 i} x_{1}(k)+E_{2 i} p(k)+E_{3 i} w(k)\right\|^{2}
$$

in the discrete-time case with $T \rightarrow \infty$ and $i=1, \ldots, s$. Note that $T$ is an integer in the discrete-time case. $A_{1}, A_{21}, A_{2}, B_{1}, B_{2}, C_{1}, C_{2}, D$ and $L$ are known constant matrices that represent the nominal system, $A_{2}$ may be an unstable matrix. $H_{11 i}, H_{12 i}, H_{2 i}, E_{11 i}, E_{2 i}$ and $E_{3 i}$ are known constant matrices describing how the uncertainties affect the nominal system.

In this paper, the problem is to estimate the vector $z(t)$ from the measurements $y(t)$.

For this purpose, consider a functional filter described by the following state-space representation

$$
\begin{aligned}
\sigma \eta & =N \eta+J y \\
\widehat{z} & =\eta+E y
\end{aligned}
$$

where $\widehat{z}(t) \in \mathbb{R}^{r}$ is the estimate of $z(t)$. This filter differs from that of Watson and Grigoriadis (1998) and has the form given in Darouach (2000); Darouach et al. (2001). Matrices $N, J$ and $E$ are of appropriate dimensions. From remark 3.1 in de Souza (2000), we note an interest to consider a proper filter i.e. a filter with direct feedforward (matrix $E$ ) from $y$ to $\widehat{z}$. Generally, the smallest level of disturbance attenuation that can be achieved with a strictly proper filter is larger than that achieved with a proper filter.

Before proceeding, let us make the following assumption on system (1) (or (5)).

\section{Assumption 1.}

(i) The sub-system (1a) is quadratically stable Li and Fu (1997); Xie et al. (1994); Xie (1996).

(ii) Matrix $A_{2}$ may be unstable.

Let the filtering error be given by

$$
e=z-\widehat{z}
$$

then

$$
e=L x-\widehat{z}=\varepsilon-E H_{2} p-E D w
$$

where

$$
\begin{aligned}
\varepsilon & =\Psi x-\eta \\
\Psi & =L-E C .
\end{aligned}
$$

From (10), notice that the time derivative of the error $e(t)$ is a function of the time derivative of the disturbances $w(t)$. To avoid the use of $\sigma w(t)$ in the dynamics of the error $e(t)$, consider $\varepsilon(t)$ as a new "state vector". Then the transfer function $T_{w e}$ from $w(t)$ to $e(t)$ has the following state space realization

$$
\left\{\begin{array}{l}
\sigma \varepsilon=N \varepsilon+(\Psi A-N \Psi-J C) x+\left(\Psi H_{1}-J H_{2}\right) p+(\Psi B-J D) w \\
e=\varepsilon-E H_{2} p-E D w .
\end{array}\right.
$$

As in Watson and Grigoriadis (1998), the filter is said to be unbiased if the error dynamics is independent of the system state $x$ in the nominal case.

The problem of the robust unbiased functional $\mathcal{H}_{\infty}$ filtering can now be stated as follows. 
Problem 1. Determine the filter matrices $N, J$ and $E$ (with $\Psi=L-E C$ ) such that

(i) the filter (8) is unbiased,

(ii) the filtering error (13) is stable and a given $\gamma>0$ is an upper bound to the $\mathcal{L}_{2}-\mathcal{L}_{2}$ gain with zero initial condition, i.e.

$$
\sup _{w \in \mathcal{L}_{2}} \frac{\|e\|_{\mathcal{L}_{2}}}{\|w\|_{\mathcal{L}_{2}}}<\gamma,\|w\|_{\mathcal{L}_{2}} \neq 0
$$

where assumption 1 holds and the dynamics of the estimation error $e$ is given by relation (13) for any admissible IQC (see (6) or (7)).

\subsection{On the interest of the unbiasedness in filtering}

We show here the meaning of the biased and the unbiased filters in robust filtering for uncertain continuous-time systems (the discrete-time case is similar). For simplicity, consider the following system with norm-bounded uncertainties

$$
\begin{aligned}
\sigma x & =\left(A+\Delta_{A}(t)\right) x+B w \\
y & =\left(C+\Delta_{C}(t)\right) x+D w \\
z & =L x
\end{aligned}
$$

where

$$
\left[\begin{array}{c}
\Delta_{A}(t) \\
\Delta_{C}(t)
\end{array}\right]=\left[\begin{array}{c}
H_{1} \\
H_{2}
\end{array}\right]\left(I_{k_{1}}-\Delta(t) E_{2}\right)^{-1} \Delta(t) E_{11} \quad \text { and } \quad\|\Delta(t)\| \leqslant 1
$$

Notice that system (15) can be rewritten in the following IQC form (see (5))

$$
\begin{aligned}
\sigma x & =A x+H_{1} p+B w \\
y & =C x+H_{2} p+D w \\
z & =L x
\end{aligned}
$$

where $s=1$. The fictituous output $q$ is given by

$$
q=E_{11} x(t)+E_{2} p(t)
$$

with $p=\Delta(t) q$.

From (16), the uncertain variable $p$ of (17) satisfies the following IQC

$$
\int_{0}^{T}\|p(t)\|^{2} \mathrm{~d} t \leqslant \int_{0}^{T}\left\|E_{11} x(t)+E_{2} p(t)\right\| \mathrm{d} t
$$

Then the estimation error $e(t)=z(t)-\widehat{z}(t)$ has the following uncertain dynamics (see (13))

$$
\left\{\begin{array}{l}
\sigma \varepsilon=N \varepsilon+\underbrace{(\Psi A-N \Psi-J C)}_{=\Phi} x+\left(\Psi \Delta_{A}(t)-J \Delta_{C}(t)\right) x+(\Psi B-J D) w \\
e=\varepsilon-E \Delta_{C}(t) x-E D w .
\end{array}\right.
$$

If $\Phi=0$ (this relation is also called the Sylvester equation (see (21))), then the estimation error $e(t)$ is unbiased in the nominal case $\left(\Delta_{A}(t)=0\right.$ and $\left.\Delta_{C}(t)=0\right)$ since its dynamics does not depend on the state $x(t)$. In this case, if matrix $N$ is stable, the estimation error $e(t)$ converges asymptotically to zero even if matrix $A$ is unstable. This is not the case when $\Phi \neq 0$ (i.e. if the Sylvester equation is not satisfied), and, in this case, the filter is a biased one. Thus, it is obvious that in the nominal case the unbiased filter has a better behaviour than the biased one.

Consider now the case where there are uncertainties in the system $\left(\Delta_{A}(t) \neq 0\right.$ and $\left.\Delta_{C}(t) \neq 0\right)$. Then the error dynamics (20) has an additional term depending on $x(t)$ and matrices $\Psi, N$ and $J$ must be chosen such that the term $\Phi x(t)$ in (20) fights against the effect of $x(t)$ introduced by the uncertainties through 
$\left(\Psi \Delta_{A}(t)-J \Delta_{C}(t)\right) x(t)$. The ideal case is when $\Phi=-\left(\Psi \Delta_{A}(t)-J \Delta_{C}(t)\right)$, thus the estimation error $e(t)$ will not have any term depending explicitely on $x(t)$ in $(20)$. This situation can not be guaranteed as the uncertainties are unknown. But, the unbiased filter can still be used in the uncertain case as it will be the best one in the nominal case and may reduce the effect of $x(t)$ on the error dynamics even in the uncertain case. Moreover, the unbiasedness condition will permit to parameterize all the filter matrices through a single gain matrix in the sequel and to consider an unstable matrix $A_{2}$ as in item (ii) of assumption 1.

\subsection{Derivation of the unbiased functional filter conditions}

The unbiasedness of the filter (8) is achieved if and only if the following Sylvester equation

$$
\Psi A-N \Psi-J C=0
$$

holds O'Reilly (1983), while the stability requirement of the filter is satisfied if the matrix $N$ is Hurwitz.

Since matrix $L$ is of full row rank, equation (21) is equivalent to

$$
\begin{aligned}
0 & =\Psi A L^{\dagger}-N \Psi L^{\dagger}-J C L^{\dagger} \\
0 & =\Psi \bar{A}+N E \bar{C}-J \bar{C}
\end{aligned}
$$

with

$$
\begin{aligned}
& \bar{A}=A\left(I_{n}-L^{\dagger} L\right), \\
& \bar{C}=C\left(I_{n}-L^{\dagger} L\right) .
\end{aligned}
$$

Using the definition of $\Psi$, relation (22a) can be rewritten as

$$
N=\overline{\bar{A}}-\mathcal{K} \overline{\bar{C}}
$$

where

$$
\begin{aligned}
& \overline{\bar{A}}=L A L^{\dagger}, \\
& \overline{\bar{C}}=\left[\begin{array}{c}
C A L^{\dagger} \\
C L^{\dagger}
\end{array}\right], \\
& \mathcal{K}=\left[\begin{array}{ll}
E & K
\end{array}\right] \text { with } K=J-N E .
\end{aligned}
$$

Then using relations (12) and (25c), equation (22b) can be rewritten as

$$
\mathcal{K} \Sigma=\left[\begin{array}{ll}
E & K
\end{array}\right] \Sigma=L \bar{A}
$$

where

$$
\Sigma=\left[\begin{array}{c}
C \bar{A} \\
\bar{C}
\end{array}\right],
$$

and a general solution to equation (26), if it exists, is given by

$$
\left[\begin{array}{ll}
E & K
\end{array}\right]=L \bar{A} \Sigma^{\dagger}+Z\left(I_{2 p}-\Sigma \Sigma^{\dagger}\right) .
$$

where $Z$ is an arbitrary matrix of appropriate dimensions.

From the definition of $\Psi$ and using (25) and (28), relation (24) can be rewritten as

$$
N=\widetilde{A}-Z \widetilde{C}
$$

where

$$
\begin{aligned}
\widetilde{A} & =\overline{\bar{A}}-L \bar{A} \Sigma^{\dagger} \overline{\bar{C}} \\
\widetilde{C} & =\left(I_{2 p}-\Sigma \Sigma^{\dagger}\right) \overline{\bar{C}} .
\end{aligned}
$$


The transfer function $T_{w e}$ from $w(t)$ to $e(t)$, given by (13), becomes

$$
\left\{\begin{array}{c}
\sigma \varepsilon=(\widetilde{A}-Z \widetilde{C}) \varepsilon+\left(L H_{1}-\widetilde{A} E H_{2}-E C H_{1}-K H_{2}+Z \widetilde{C} E H_{2}\right) p \\
+(L B-\widetilde{A} E D-E C B-K D+Z \widetilde{C} E D) w \\
e=\varepsilon-E H_{2} p-E D w
\end{array}\right.
$$

where $E$ and $K$ are given by (28).

In system (31), the filtering error is bilinear in the gain parameter $Z$ due to the product $Z \widetilde{C} E$. This bilinearity is intrinsically linked to the unbiasedness condition (21). Indeed, the "bilinearity" $N \Psi$ in (21) yields a gain $K$ (see $(25 \mathrm{c})$ ) containing the product $N E$. In order to avoid this kind of "bilinearity", we consider the following constraint

$$
E\left[\begin{array}{ll}
H_{2} & D
\end{array}\right]=0 .
$$

Adding the constraint $E\left[\begin{array}{ll}H_{2} & D\end{array}\right]=0$, relations (26), (27) and (28) become

$$
\begin{aligned}
{\left[\begin{array}{ll}
E & K
\end{array}\right] \widehat{\Sigma} } & =\left[\begin{array}{lll}
0 & 0 & L \bar{A}
\end{array}\right] \\
\widehat{\Sigma} & =\left[\begin{array}{ccc}
H_{2} & D & C \bar{A} \\
0 & 0 & \bar{C}
\end{array}\right] \\
{\left[\begin{array}{ll}
E & K
\end{array}\right] } & =\left[\begin{array}{lll}
0 & 0 & L \bar{A}
\end{array}\right] \widehat{\Sigma}^{\dagger}+Z\left(I_{2 p}-\widehat{\Sigma} \widehat{\Sigma}^{\dagger}\right)
\end{aligned}
$$

with $K=J-N E$.

Notice that with $E\left[\begin{array}{ll}H_{2} & D\end{array}\right]=0$, the transfer function $T_{w e}$ from $w(t)$ to $e(t)$, given by (31), has no feedthrough term and becomes

$$
\sigma e=\left(\widehat{F}_{1}-Z \widehat{F}_{2}\right) e+\left(\widehat{M}_{1}-Z \widehat{M}_{2}\right) p+\left(\widehat{G}_{1}-Z \widehat{G}_{2}\right) w
$$

where

$$
\begin{array}{ll}
\widehat{F}_{1}=\overline{\bar{A}}-\left[\begin{array}{lll}
0 & 0 & L \bar{A}
\end{array}\right] \widehat{\Sigma}^{\dagger} \overline{\bar{C}}, & \widehat{F}_{2}=\left(I_{2 p}-\widehat{\Sigma} \widehat{\Sigma}^{\dagger}\right) \overline{\bar{C}} \\
\widehat{G}_{1}=L B-\left[\begin{array}{lll}
0 & 0 & L \bar{A}
\end{array}\right] \widehat{\Sigma}^{\dagger}\left[\begin{array}{c}
C B \\
D
\end{array}\right], & \widehat{G}_{2}=\left(I_{2 p}-\widehat{\Sigma} \widehat{\Sigma}^{\dagger}\right)\left[\begin{array}{c}
C B \\
D
\end{array}\right], \\
\widehat{M}_{1}=L H_{1}-\left[\begin{array}{lll}
0 & 0 & L \bar{A}
\end{array}\right] \widehat{\Sigma}^{\dagger}\left[\begin{array}{c}
C H_{1} \\
H_{2}
\end{array}\right], & \widehat{M}_{2}=\left(I_{2 p}-\widehat{\Sigma} \widehat{\Sigma}^{\dagger}\right)\left[\begin{array}{c}
C H_{1} \\
H_{2}
\end{array}\right] .
\end{array}
$$

From relations (33a), the necessary and sufficient condition for the existence of an unbiased filter (8) verifying $E\left[\begin{array}{ll}H_{2} & D\end{array}\right]=0$ is given by the following theorem.

Theorem 1. Under constraint (32), the unbiasedness of filter (8) for system (1) (or (5)) is guaranteed if and only if

$$
\operatorname{rank}\left[\begin{array}{ccc}
0 & 0 & L A \\
H_{2} & D & C A \\
0 & 0 & C \\
0 & 0 & L
\end{array}\right]=\operatorname{rank}\left[\begin{array}{ccc}
H_{2} & D & C A \\
0 & 0 & C \\
0 & 0 & L
\end{array}\right]
$$

Proof. From the linear algebra theory Lancaster and Tismenetsky (1985), there exists a solution $\left[\begin{array}{ll}E & K\end{array}\right]$ to equation (33a) if and only if

$$
\left[\begin{array}{lll}
0 & 0 & L \\
A
\end{array}\right]\left(I_{n+2 p}-\widehat{\Sigma}^{\dagger} \widehat{\Sigma}\right)=0
$$

or if and only if

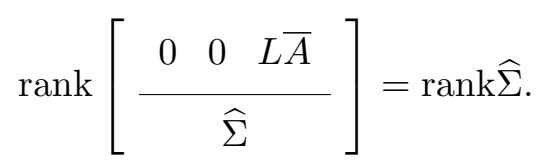

Then, right-multiplying each side of equation (36) by $\left[\begin{array}{cccc}I_{k} & 0 & 0 & 0 \\ 0 & I_{m} & 0 & 0 \\ 0 & 0 & L^{\dagger} & I_{n}-L^{\dagger} L\end{array}\right]$ gives (37). 
Remark 1. The case $E=0$ in the filter equation (8), considered in Watson and Grigoriadis (1998), is a special case. Indeed, if $E=0$, equations (25c) and (33b) become $\mathcal{K}=K=J$ and $\Sigma=\bar{C}$, respectively. Then the unbiasedness condition is given by

$$
\operatorname{rank}\left[\begin{array}{c}
L A \\
C \\
L
\end{array}\right]=\operatorname{rank}\left[\begin{array}{c}
C \\
L
\end{array}\right] .
$$

This condition is contained in that of theorem 1 . So, it is interesting to consider the general case where $E \neq 0$ for a global approach.

According to the previous developments, the augmented state vector $\xi$ given by

$$
\xi=\left[\begin{array}{l}
x \\
e
\end{array}\right]=\left[\begin{array}{c}
x_{1} \\
x_{2} \\
\hline e
\end{array}\right]
$$

has the following uncertain dynamics (see (1))

$$
\left\{\begin{array}{l}
\sigma \xi=\left[\begin{array}{ccc}
A_{1} & 0 & 0 \\
A_{21} & A_{2} & 0 \\
0 & 0 & N
\end{array}\right] \xi+\left[\begin{array}{c}
H_{11} \\
H_{12} \\
\left(\Psi H_{1}-J H_{2}\right)
\end{array}\right] p+\left[\begin{array}{c}
B_{1} \\
B_{2} \\
(\Psi B-J D)
\end{array}\right] w \\
q=\left[\begin{array}{lll}
E_{11} & 0 & 0
\end{array}\right] \xi+E_{2} p+E_{3} w \\
e=\left[\begin{array}{lll}
0 & 0 & I_{r}
\end{array}\right] \xi
\end{array}\right.
$$

where the matrix $\Psi$ given by (see (12))

$$
\Psi=\left[\begin{array}{ll}
\Psi_{1} & \Psi_{2}
\end{array}\right]=\left[\begin{array}{ll}
L_{1} & L_{2}
\end{array}\right]-E\left[\begin{array}{ll}
C_{1} & C_{2}
\end{array}\right]
$$

satisfies the unbiasedness condition (21) for the nominal system (1) with $H_{1}=0$ and $H_{2}=0$, i.e.

$$
\left[\begin{array}{ll}
\Psi_{1} & \Psi_{2}
\end{array}\right]\left[\begin{array}{cc}
A_{1} & 0 \\
A_{21} & A_{2}
\end{array}\right]-N\left[\begin{array}{ll}
\Psi_{1} & \Psi_{2}
\end{array}\right]-J\left[\begin{array}{ll}
C_{1} & C_{2}
\end{array}\right]=0
$$

\section{Robust reduced order unbiased $\mathcal{H}_{\infty}$ filtering}

This section is dedicated to the design of a robust functional unbiased filter.

Here, we assumed that the existence condition of filter (8) is achivied i.e. relation (36) is verified.

Now,from (40), let us introduce the following parametrized system

$$
\left\{\begin{aligned}
\sigma \widehat{\xi} & =\left[\begin{array}{ccc}
A_{1} & 0 & 0 \\
A_{21} & A_{2} & 0 \\
0 & 0 & N
\end{array}\right] \widehat{\xi}+\left[\begin{array}{c}
H_{11} \Pi^{-1 / 2} \\
H_{12} \Pi^{-1 / 2} \\
\left(\Psi H_{1}-J H_{2}\right) \Pi^{-1 / 2}
\end{array}\right] p+\left[\begin{array}{c}
\gamma^{-1} B_{1} \\
\gamma^{-1} B_{2} \\
\gamma^{-1}(\Psi B-J D)
\end{array}\right] w \\
q & =\left[\begin{array}{lll}
\Pi^{1 / 2} E_{11} & 0 & 0
\end{array}\right] \widehat{\xi}+\Pi^{1 / 2} E_{2} \Pi^{-1 / 2} p+\gamma^{-1} \Pi^{1 / 2} E_{3} w \\
e & =\left[\begin{array}{lll}
0 & 0 & I_{r}
\end{array}\right] \widehat{\xi}
\end{aligned}\right.
$$

where $\gamma \in \mathbb{R}^{+}$is given and

$$
q=\left[\begin{array}{lll}
q_{1}^{T} & \cdots & q_{s}^{T}
\end{array}\right]^{T}
$$

is a fictitious output, and

$$
\Pi=\operatorname{bdiag}\left\{\mu_{1} I_{k_{1}}, \cdots, \mu_{s} I_{k_{s}}\right\}
$$

where the $\mu_{i}>0, i=1,2, \cdots, s$, are scaling parameters to be chosen and bdiag(.) represents a blockdiagonal matrix. Note that $\Pi \in \mathbb{R}^{\bar{k} \times \bar{k}}$ with $\bar{k}=\sum_{i=1}^{s} k_{i}$.

Using this parametrized system and problem 1, let us give the following lemma. 
Lemma 1. Under assumption 1 and assuming that relation (36) is verified, the filter (8) solves the robust $\mathcal{H}_{\infty}$ filtering stated in problem 1 for any admissible IQC (see (6) or (7)) if, for a given $\gamma>0$, there exists $\Pi>0$ given by (45) such that the system (8) is a $\gamma$-suboptimal $\mathcal{H}_{\infty}$ stable unbiased functional filter for the parametrized system (43) achieving the following $\mathcal{L}_{2}-\mathcal{L}_{2}$ gain

$$
\sup _{\left[\begin{array}{ll}
p^{T} & w^{T}
\end{array}\right]^{T} \in \mathcal{L}_{2}} \frac{\left\|\left[\begin{array}{ll}
q^{T} & e^{T}
\end{array}\right]^{T}\right\|_{\mathcal{L}_{2}}}{\left\|\left[\begin{array}{ll}
p^{T} & w^{T}
\end{array}\right]^{T}\right\|_{\mathcal{L}_{2}}}<1,\left\|\left[\begin{array}{c}
p \\
w
\end{array}\right]\right\|_{\mathcal{L}_{2}} \neq 0
$$

Proof. From relation (36) and using (1), (41) and (42), the dynamics of the state vector $\xi(t)$ defined in (39) is given by system (40) which is associated to the parametrized system (43).

Since the filter (8) is unbiased, then relation (21) holds. As the existence and stability conditions of the filter (8) are assumed to be fulfilled, there exists a stable matrix $N$ which is solution to the unbiasedness equation (21). By rewriting this relation as (42), we have

$$
\Psi_{2} A_{2}-N \Psi_{2}-J C_{2}=0 .
$$

Recall that $A_{2}$ may be unstable. Then in systems (40) and (43), the unstable subspace spanned by $\left[\begin{array}{lll}0 & x_{2}^{T}(t) & 0\end{array}\right]^{T}$ has no effect on the outputs $q(t)$ and $e(t)$. To evaluate the induced gain given by

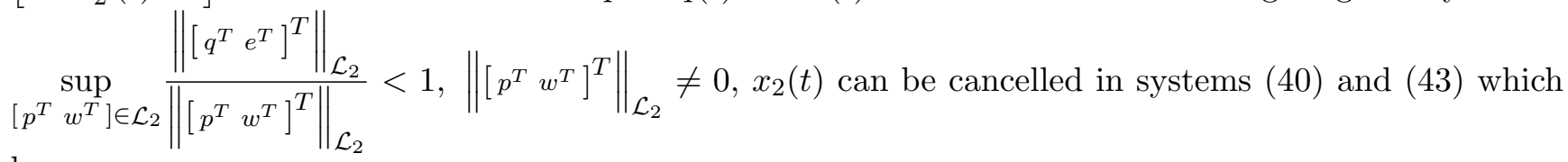
become

$$
\left\{\begin{array}{l}
\sigma \bar{\xi}=\left[\begin{array}{cc}
A_{1} & 0 \\
0 & N
\end{array}\right] \bar{\xi}+\left[\begin{array}{c}
H_{11} \\
\left(\Psi H_{1}-J H_{2}\right)
\end{array}\right] p+\left[\begin{array}{c}
B_{1} \\
(\Psi B-J D)
\end{array}\right] w \\
q=\left[\begin{array}{ll}
E_{11} & 0
\end{array}\right] \bar{\xi}+E_{2} p+E_{3} w \\
e=\left[\begin{array}{ll}
0 & I_{r}
\end{array}\right] \bar{\xi}
\end{array}\right.
$$

and

$$
\left\{\begin{aligned}
\dot{\bar{\xi}} & =\left[\begin{array}{cc}
A_{1} & 0 \\
0 & N
\end{array}\right] \widehat{\bar{\xi}}+\left[\begin{array}{c}
H_{11} \Pi^{-1 / 2} \\
\left(\Psi H_{1}-J H_{2}\right) \Pi^{-1 / 2}
\end{array}\right] p+\left[\begin{array}{c}
\gamma^{-1} B_{1} \\
\gamma^{-1}(\Psi B-J D)
\end{array}\right] w \\
q & =\left[\begin{array}{ll}
\Pi^{1 / 2} E_{11} & 0
\end{array}\right] \overline{\bar{\xi}}+\Pi^{1 / 2} E_{2} \Pi^{-1 / 2} p+\gamma^{-1} \Pi^{1 / 2} E_{3} w \\
e & =\left[\begin{array}{ll}
0 & I_{r}
\end{array}\right] \widehat{\widehat{\xi}}
\end{aligned}\right.
$$

respectively, where $\widehat{\bar{\xi}}(t)$ is of the same dimension as $\bar{\xi}(t)$ and

$$
\bar{\xi}=\left[\begin{array}{ll}
x_{1}^{T} & e^{T}
\end{array}\right]^{T} .
$$

Since the uncertain subsystem given by (1a) is quadratically stable from assumption 1 , the lemma is proved by applying theorem 3 and theorem 4 of $\mathrm{Li}$ and $\mathrm{Fu}(1997)$.

Note that relation (49) can be rewritten as

$$
\left\{\begin{array}{l}
\sigma \bar{\xi}=\left[\begin{array}{cc}
A_{1} & 0 \\
0 & \left(\widehat{F}_{1}-Z \widehat{F}_{2}\right)
\end{array}\right] \bar{\xi}+\left[\begin{array}{cc}
H_{11} \Pi^{-1 / 2} & \gamma^{-1} B_{1} \\
\left(\widehat{M}_{1}-Z \widehat{M}_{2}\right) \Pi^{-1 / 2} & \gamma^{-1}\left(\widehat{G}_{1}-Z \widehat{G}_{2}\right)
\end{array}\right] \bar{w} \\
\bar{z}=\left[\begin{array}{cc}
\Pi^{1 / 2} E_{11} & 0 \\
0 & I_{r}
\end{array}\right] \bar{\xi}+\left[\begin{array}{cc}
\Pi^{1 / 2} E_{2} \Pi^{-1 / 2} & \gamma^{-1} \Pi^{1 / 2} E_{3} \\
0 & 0
\end{array}\right] \bar{w}
\end{array}\right.
$$

where

$$
\bar{z}=\left[\begin{array}{ll}
q^{T} & e^{T}
\end{array}\right]^{T} \quad \text { and } \quad \bar{w}=\left[\begin{array}{ll}
p^{T} & w^{T}
\end{array}\right]^{T} .
$$

In system (51), the determination of the gain matrix $Z$ can be transformed into the following static output feedback control problem

$$
\left\{\begin{array}{l}
\sigma \bar{\xi}=\mathbb{A} \bar{\xi}+\mathbb{B}_{\bar{w}} \bar{w}+\mathbb{B}_{\bar{u}} \bar{u} \\
\bar{z}=\mathbb{C}_{\bar{z}} \bar{\xi}+\mathbb{D}_{\bar{z}} \bar{w} \bar{w} \\
\bar{y}=\mathbb{C}_{\bar{y}} \bar{\xi}+\mathbb{D}_{\bar{y}} \bar{w} \bar{w}
\end{array}\right.
$$


with

$$
\bar{u}=-Z \bar{y}
$$

where $Z$ is the static output feedback controller to be designed in order to achieve stability and the attenuation from the perturbation $\bar{w}(t)$ to the controlled output $\bar{z}(t)$. The vectors $\bar{u}(t)$ and $\bar{y}(t)$ are the "control input" and the "measured output", respectively. The matrices and the vectors introduced in (53) are given by

$$
\begin{aligned}
& \mathbb{A}=\left[\begin{array}{cc}
A_{1} & 0 \\
0 & \widehat{F}_{1}
\end{array}\right], \\
& \mathbb{B}_{\bar{w}}=\left[\begin{array}{ll}
H_{11} \Pi^{-1 / 2} & \gamma^{-1} B_{1} \\
\widehat{M}_{1} \Pi^{-1 / 2} & \gamma^{-1} \widehat{G}_{1}
\end{array}\right], \\
& \mathbb{B}_{\bar{u}}=\left[\begin{array}{c}
0 \\
I_{r}
\end{array}\right], \\
& \mathbb{C}_{\bar{z}}=\left[\begin{array}{cc}
\Pi^{1 / 2} E_{11} & 0 \\
0 & I_{r}
\end{array}\right], \\
& \mathbb{D}_{\bar{z} \bar{w}}=\left[\begin{array}{cc}
\Pi^{1 / 2} E_{2} \Pi^{-1 / 2} & \gamma^{-1} \Pi^{1 / 2} E_{3} \\
0 & 0
\end{array}\right] \\
& \mathbb{C}_{\bar{y}}=\left[\begin{array}{ll}
0 & \widehat{F}_{2}
\end{array}\right] \text {, } \\
& \mathbb{D}_{\bar{y} \bar{w}}=\left[\widehat{M}_{2} \Pi^{-1 / 2} \quad \gamma^{-1} \widehat{G}_{2}\right] \text {. }
\end{aligned}
$$

Before proceeding, let us decompose some of the above matrices as follows

$$
\begin{aligned}
& \mathbb{B}_{\bar{w}}=\left[\begin{array}{ll}
\mathbb{B}_{p} \Pi^{-1 / 2} & \gamma^{-1} \mathbb{B}_{w}
\end{array}\right] \text { with } \mathbb{B}_{p}=\left[\begin{array}{l}
H_{11} \\
\widehat{M}_{1}
\end{array}\right] \text { and } \mathbb{B}_{w}=\left[\begin{array}{l}
B_{1} \\
\widehat{G}_{1}
\end{array}\right] \\
& \mathbb{C}_{\bar{z}}=\left[\begin{array}{c}
\Pi^{1 / 2} \mathbb{C}_{q} \\
\mathbb{C}_{e}
\end{array}\right] \text { with } \mathbb{C}_{q}=\left[\begin{array}{ll}
E_{11} & 0
\end{array}\right] \text { and } \mathbb{C}_{e}=\left[\begin{array}{ll}
0 & I_{r}
\end{array}\right] \\
& \mathbb{D}_{\bar{z} \bar{w}}=\left[\begin{array}{cc}
\Pi^{1 / 2} \mathbb{D}_{q p} \Pi^{-1 / 2} & \gamma^{-1} \Pi^{1 / 2} \mathbb{D}_{q w} \\
\mathbb{D}_{e p} \Pi^{-1 / 2} & \gamma^{-1} \mathbb{D}_{e w}
\end{array}\right] \text { with } \mathbb{D}_{q p}=E_{2}, \mathbb{D}_{q w}=E_{3} \text { and } \mathbb{D}_{e p}=\mathbb{D}_{e w}=0, \\
& \mathbb{D}_{\bar{y} \bar{w}}=\left[\begin{array}{ll}
\mathbb{D}_{y p} \Pi^{-1 / 2} & \gamma^{-1} \mathbb{D}_{y w}
\end{array}\right] \text { with } \mathbb{D}_{y p}=\widehat{M}_{2} \text { and } \mathbb{D}_{y w}=\widehat{G}_{2} \text {. }
\end{aligned}
$$

In order to unify the notations, we also put $\mathbb{B}_{u}=\mathbb{B}_{\bar{u}}$ and $\mathbb{C}_{y}=\mathbb{C}_{\bar{y}}$.

Then the following theorems 2 and 3 give the solution, in terms of the gain matrix $Z$, to the robust $\mathcal{H}_{\infty}$ filtering problem in the continuous and discrete-time cases, respectively.

Theorem 2 (Robust functional $\mathcal{H}_{\infty}$ filtering : continuous-time case). Consider that assumption 1 holds. Under relation (36), there exists a continuous-time $\gamma$-suboptimal robust $\mathcal{H}_{\infty}$ functional unbiased filter (8) for the uncertain system (1) if there exist matrices $\mathbb{P}=\mathbb{P}^{T}>0$ and $\mathbb{Q}=\mathbb{Q}^{T}>0, \mathbb{P}, \mathbb{Q} \in \mathbb{R}^{\left(n_{1}+r\right) \times\left(n_{1}+r\right)}$ such that

$$
\begin{aligned}
& \Upsilon_{c 1}=\left[\begin{array}{c|c}
\mathbb{K}_{y} & 0 \\
\hline 0 & I_{\bar{k}+r}
\end{array}\right]^{T}\left[\begin{array}{ccc|cc}
\mathbb{A}^{T} \mathbb{P}+\mathbb{P} \mathbb{P} & \mathbb{P B}_{p} & \mathbb{P B}_{w} & \mathbb{C}_{q}^{T} \Pi & \mathbb{C}_{e}^{T} \\
\mathbb{B}_{p}^{T} \mathbb{P} & -\Pi & 0 & \mathbb{D}_{q p}^{T} \Pi & \mathbb{D}_{e p}^{T} \\
\mathbb{B}_{w}^{T} \mathbb{P} & 0 & -\gamma^{2} I_{m} & \mathbb{D}_{q w}^{T} \Pi \mathbb{D}_{e w}^{T} \\
\hline \Pi \mathbb{C}_{q} & \Pi \mathbb{D}_{q p} & \Pi \mathbb{D}_{q w} & -\Pi & 0 \\
\mathbb{C}_{e} & \mathbb{D}_{e p} & \mathbb{D}_{e w} & 0 & -I_{r}
\end{array}\right]\left[\begin{array}{c|c}
\mathbb{K}_{y} & 0 \\
\hline 0 & I_{\bar{k}+r}
\end{array}\right]<0, \\
& \Upsilon_{c 2}=\left[\begin{array}{c|c}
\mathbb{K}_{u} & 0 \\
\hline 0 & I_{\bar{k}+m}
\end{array}\right]^{T}\left[\begin{array}{ccc|cc}
\mathbb{Q A}^{T}+\mathbb{A} \mathbb{Q} & \mathbb{Q}_{q}^{T} & \mathbb{Q C}_{e}^{T} & \mathbb{B}_{p} \Pi^{-1} & \mathbb{B}_{w} \\
\mathbb{C}_{q} \mathbb{Q} & -\Pi^{-1} & 0 & \mathbb{D}_{q p} \Pi^{-1} & \mathbb{D}_{q w} \\
\mathbb{C}_{e} \mathbb{Q} & 0 & -I_{r} & \mathbb{D}_{e p} \Pi^{-1} & \mathbb{D}_{e w} \\
\hline \Pi^{-1} \mathbb{B}_{p}^{T} & \Pi^{-1} \mathbb{D}_{q p}^{T} \Pi^{-1} \mathbb{D}_{e p}^{T} & -\Pi^{-1} & 0 \\
\mathbb{B}_{w}^{T} & \mathbb{D}_{q w}^{T} & \mathbb{D}_{e w}^{T} & 0 & -\gamma^{2} I_{m}
\end{array}\right]\left[\begin{array}{c|c}
\mathbb{K}_{u} & 0 \\
\hline 0 & I_{\bar{k}+m}
\end{array}\right]<0, \\
& I_{n_{1}+r}=\mathbb{P} \mathbb{Q},
\end{aligned}
$$

where $\mathbb{K}_{y}$ and $\mathbb{K}_{u}$ are two matrices whose columns span the null spaces of $\left[\begin{array}{llll}\mathbb{C}_{y} & \mathbb{D}_{y p} & \mathbb{D}_{y w}\end{array}\right]$ and $\left[\begin{array}{lll}\mathbb{B}_{u}^{T} & 0 & 0\end{array}\right]$, respectively. All gains $Z$ are given by

$$
Z=\overline{\mathbb{B}}_{R}^{\dagger} \mathbb{K} \overline{\mathbb{C}}_{L}^{\dagger}+\mathbb{Z}-\overline{\mathbb{B}}_{R}^{\dagger} \overline{\mathbb{B}}_{R} \mathbb{Z} \overline{\mathbb{C}}_{L} \overline{\mathbb{C}}_{L}^{\dagger}
$$


where

$$
\begin{aligned}
& \mathbb{K}=-\mathbb{R}_{1}^{-1} \overline{\mathbb{B}}_{L}^{T} \mathbb{S}_{1} \overline{\mathbb{C}}_{R}^{T}\left(\overline{\mathbb{C}}_{R} \mathbb{S}_{1} \overline{\mathbb{C}}_{R}^{T}\right)^{-1}+\mathbb{R}_{1}^{-1} \mathbb{S}_{2}^{1 / 2} \mathbb{R}_{2}\left(\overline{\mathbb{C}}_{R} \mathbb{S}_{1} \overline{\mathbb{C}}_{R}^{T}\right)^{-1 / 2} \\
& \mathbb{S}_{1}=\left(\overline{\mathbb{B}}_{L} \mathbb{R}_{1}^{-1} \overline{\mathbb{B}}_{L}^{\dagger}-\overline{\mathbb{Q}}\right)^{-1}>0 \\
& \mathbb{S}_{2}=\mathbb{R}_{1}-\overline{\mathbb{B}}_{L}^{T}\left(\mathbb{S}_{1}-\mathbb{S}_{1} \overline{\mathbb{C}}_{R}^{T}\left(\overline{\mathbb{C}}_{R} \mathbb{S}_{1} \overline{\mathbb{C}}_{R}^{T}\right)^{-1} \overline{\mathbb{C}}_{R} \mathbb{S}_{1}\right) \overline{\mathbb{B}}_{L} \\
& {\left[\begin{array}{cc}
\overline{\mathbb{B}} & \overline{\mathbb{Q}} \\
\bullet & \overline{\mathbb{C}}
\end{array}\right]=\left[\begin{array}{c|ccccc}
-\mathbb{B}_{u} & \mathbb{A} \mathbb{Q}+\mathbb{Q}^{T} & \mathbb{Q}_{q}^{T} & \mathbb{Q C}_{e}^{T} & \mathbb{B}_{p} \Pi^{-1} & \mathbb{B}_{w} \\
0 & \mathbb{C}_{q} \mathbb{Q} & -\Pi^{-1} & 0 & \mathbb{D}_{q p} \Pi^{-1} & \mathbb{D}_{q w} \\
0 & \mathbb{C}_{e} \mathbb{Q} & 0 & -I_{r} & \mathbb{D}_{e p} \Pi^{-1} & \mathbb{D}_{e w} \\
0 & \Pi^{-1} \mathbb{B}_{p}^{T} & \Pi^{-1} \mathbb{D}_{q p}^{T} \Pi^{-1} \mathbb{D}_{e p}^{T}-\Pi^{-1} & 0 \\
0 & \mathbb{B}_{w}^{T} & \mathbb{D}_{q w}^{T} & \mathbb{D}_{e w}^{T} & 0 & -\gamma^{2} I_{m} \\
\hline \bullet & \mathbb{C}_{y} \mathbb{Q} & 0 & 0 & \mathbb{D}_{y p} \Pi^{-1} & \mathbb{D}_{y w}
\end{array}\right]}
\end{aligned}
$$

and $\mathbb{R}_{1}, \mathbb{R}_{2}$ and $\mathbb{Z}$ are arbitrary matrices of appropriate dimensions satisfying $\mathbb{R}_{1}=\mathbb{R}_{1}^{T}>0$ and $\left\|\mathbb{R}_{2}\right\|<1$. Matrices $\overline{\mathbb{B}}_{L}, \overline{\mathbb{B}}_{R}, \overline{\mathbb{C}}_{L}$ and $\overline{\mathbb{C}}_{R}$ are any full rank matrices such that $\overline{\mathbb{B}}=\overline{\mathbb{B}}_{L} \overline{\mathbb{B}}_{R}$ and $\overline{\mathbb{C}}=\overline{\mathbb{C}}_{L} \overline{\mathbb{C}}_{R}$.

Proof. Recall that assumption 1 holds and relation (36) is verified. From lemma 1, problem 1 is solved if the closed-loop system (53)-(54) given by

$$
\left\{\begin{array}{l}
\sigma \bar{\xi}=\left(\mathbb{A}-\mathbb{B}_{\bar{u}} Z \mathbb{C}_{\bar{y}}\right) \bar{\xi}+\left(\mathbb{B}_{\bar{w}}-\mathbb{B}_{\bar{u}} Z \mathbb{D}_{\bar{y} \bar{w}}\right) \bar{w} \\
\bar{z}=\mathbb{C}_{\bar{z}} \bar{\xi}+\mathbb{D}_{\bar{z} \bar{w}} \bar{w}
\end{array}\right.
$$

is stable with an $\mathcal{H}_{\infty}$ norm less than 1 . From the bounded real lemma Xie (1996), this is verified if and only if there exists a matrix $\mathbb{P}=\mathbb{P}^{T}>0$ such that

$$
\left[\begin{array}{ccccc}
\mathbb{P} \overline{\mathbb{A}}+\overline{\mathbb{A}}^{T} \mathbb{P} & \mathbb{P B}_{p} \Pi^{-1 / 2} & \gamma^{-1} \mathbb{P B}_{w} & \mathbb{C}_{q}^{T} \Pi^{1 / 2} & \mathbb{C}_{e}^{T} \\
\Pi^{-1 / 2} \overline{\mathbb{B}}_{p}^{T} \mathbb{P} & -I_{\bar{k}} & 0 & \Pi^{-1 / 2} \mathbb{D}_{q p}^{T} \Pi^{1 / 2} & \Pi^{-1 / 2} \mathbb{D}_{e p}^{T} \\
\gamma^{-1} \overline{\mathbb{B}}_{w}^{T} \mathbb{P} & 0 & -I_{m} & \gamma^{-1} \mathbb{D}_{q w}^{T} \Pi^{1 / 2} & \gamma^{-1} \mathbb{D}_{e w}^{T} \\
\Pi^{1 / 2} \mathbb{C}_{q} & \Pi^{1 / 2} \mathbb{D}_{q p} \Pi^{-1 / 2} & \gamma^{-1} \Pi^{1 / 2} \mathbb{D}_{q w} & -I_{\bar{k}} & 0 \\
\mathbb{C}_{e} & \mathbb{D}_{e p} \Pi^{-1 / 2} & \gamma^{-1} \mathbb{D}_{e w} & 0 & -I_{r}
\end{array}\right]<0
$$

with

$$
\overline{\mathbb{A}}=\mathbb{A}-\mathbb{B}_{u} Z \mathbb{C}_{y}=\left[\begin{array}{cc}
A_{1} & 0 \\
0 & \widehat{F}_{1}-Z \widehat{F}_{2}
\end{array}\right], \overline{\mathbb{B}}_{p}=\mathbb{B}_{p}-\mathbb{B}_{u} Z \mathbb{D}_{y p}=\left[\begin{array}{c}
H_{11} \\
\widehat{M}_{1}-Z \widehat{M}_{2}
\end{array}\right], \overline{\mathbb{B}}_{w}=\mathbb{B}_{w}-\mathbb{B}_{u} Z \mathbb{D}_{y w}=\left[\begin{array}{c}
B_{1} \\
\widehat{G}_{1}-Z \widehat{G}_{2}
\end{array}\right] .
$$


Using $\mathbb{Q}=\mathbb{P}^{-1}$ (see (57c)), the inequality (61) is equivalent to $\left[\begin{array}{ccccc}I_{n_{1}+r} & 0 & 0 & 0 & 0 \\ 0 & 0 & 0 & I_{\bar{k}} & 0 \\ 0 & 0 & 0 & 0 & I_{r} \\ 0 & I_{\bar{k}} & 0 & 0 & 0 \\ 0 & 0 & I_{m} & 0 & 0\end{array}\right]\left[\begin{array}{ccccc}\mathbb{Q} & 0 & 0 & 0 & 0 \\ 0 & \Pi^{-1 / 2} & 0 & 0 & 0 \\ 0 & 0 & \gamma I_{m} & 0 & 0 \\ 0 & 0 & 0 & \Pi^{-1 / 2} & 0 \\ 0 & 0 & 0 & 0 & I_{r}\end{array}\right]$ $\times\left[\begin{array}{ccccc}\mathbb{P} \overline{\mathbb{A}}+\overline{\mathbb{A}}^{T} \mathbb{P} & \mathbb{P B}_{p} \Pi^{-1 / 2} & \gamma^{-1} \mathbb{P} \overline{\mathbb{B}}_{w} & \mathbb{C}_{q}^{T} \Pi^{1 / 2} & \mathbb{C}_{e}^{T} \\ \Pi^{-1 / 2} \overline{\mathbb{B}}_{p}^{T} \mathbb{P} & -I_{\bar{k}} & 0 & \Pi^{-1 / 2} \mathbb{D}_{q p}^{T} \Pi^{1 / 2} & \Pi^{-1 / 2} \mathbb{D}_{e p}^{T} \\ \gamma^{-1} \overline{\mathbb{B}}_{w}^{T} \mathbb{P} & 0 & -I_{m} & \gamma^{-1} \mathbb{D}_{q w}^{T} \Pi^{1 / 2} & \gamma^{-1} \mathbb{D}_{e w}^{T} \\ \Pi^{1 / 2} \mathbb{C}_{q} & \Pi^{1 / 2} \mathbb{D}_{q p} \Pi^{-1 / 2} & \gamma^{-1} \Pi^{1 / 2} \mathbb{D}_{q w} & -I_{\bar{k}} & 0 \\ \mathbb{C}_{e} & \mathbb{D}_{e p} \Pi^{-1 / 2} & \gamma^{-1} \mathbb{D}_{e w} & 0 & -I_{r}\end{array}\right]$ $\times\left[\begin{array}{ccccc}\mathbb{Q} & 0 & 0 & 0 & 0 \\ 0 & \Pi^{-1 / 2} & 0 & 0 & 0 \\ 0 & 0 & \gamma I_{m} & 0 & 0 \\ 0 & 0 & 0 & \Pi^{-1 / 2} & 0 \\ 0 & 0 & 0 & 0 & I_{r}\end{array}\right]\left[\begin{array}{ccccc}I_{n_{1}+r} & 0 & 0 & 0 & 0 \\ 0 & 0 & 0 & I_{\bar{k}} & 0 \\ 0 & 0 & 0 & 0 & I_{m} \\ 0 & I_{\bar{k}} & 0 & 0 & 0 \\ 0 & 0 & I_{r} & 0 & 0\end{array}\right]$ $=\underbrace{\left[\begin{array}{ccccc}\mathbb{Q A}^{T}+\mathbb{A} \mathbb{Q} & \mathbb{Q C}_{q}^{T} & \mathbb{Q}_{e}^{T} & \mathbb{B}_{p} \Pi^{-1} & \mathbb{B}_{w} \\ \mathbb{C}_{q} \mathbb{Q} & -\Pi^{-1} & 0 & \mathbb{D}_{q p} \Pi^{-1} & \mathbb{D}_{q w} \\ \mathbb{C}_{e} \mathbb{Q} & 0 & -I_{r} & \mathbb{D}_{e p} \Pi^{-1} & \mathbb{D}_{e w} \\ \Pi^{-1} B_{p}^{T} & \Pi^{-1} D_{q p}^{T} & \Pi^{-1} D_{e p}^{T} & -\Pi^{-1} & 0 \\ \mathbb{B}_{w}^{T} & \mathbb{D}_{q w}^{T} & \mathbb{D}_{e w}^{T} & 0 & -\gamma^{2} I_{m}\end{array}\right]}_{\overline{\mathbb{Q}}}$ $+\underbrace{\left[\begin{array}{c}-\mathbb{B}_{u} \\ 0 \\ 0 \\ 0 \\ 0\end{array}\right]}_{\overline{\mathbb{B}}} Z \underbrace{\left[\begin{array}{ccccc}\mathbb{C}_{y} \mathbb{Q} & 0 & 0 & \mathbb{D}_{y p} \Pi^{-1} & \mathbb{D}_{y w}\end{array}\right]}_{\overline{\mathbb{C}}}+\underbrace{\left[\begin{array}{c}\mathbb{Q C}_{y}^{T} \\ 0 \\ 0 \\ \Pi^{-1} \mathbb{D}_{y p}^{T} \\ \mathbb{D}_{y w}^{T}\end{array}\right]}_{\overline{\mathbb{C}}^{T}} Z^{T} \underbrace{\left[\begin{array}{lllll}-\mathbb{B}_{u}^{T} & 0 & 0 & 0 & 0\end{array}\right]}_{\overline{\mathbb{B}}^{T}}<0$.

The LMI (57a) and (57b) are then obtained by applying the projection lemma Iwasaki and Skelton (1994) to the above inequality, whereas equations (58) and (59) are deduced from relation (22) in Iwasaki and Skelton (1994).

The filter (8) is finally obtained by using relations (33) and (35) verifying (32).

Theorem 3 (Robust functional $\mathcal{H}_{\infty}$ filtering : discrete-time case). Consider that assumption 1 holds. Under relation (36), there exists a discrete-time $\gamma$-suboptimal robust $\mathcal{H}_{\infty}$ functional unbiased filter (8) if 
there exist matrices $\mathbb{P}=\mathbb{P}^{T}>0$ and $\mathbb{Q}=\mathbb{Q}^{T}>0, \mathbb{P}, \mathbb{Q} \in \mathbb{R}^{\left(n_{1}+r\right) \times\left(n_{1}+r\right)}$ such that

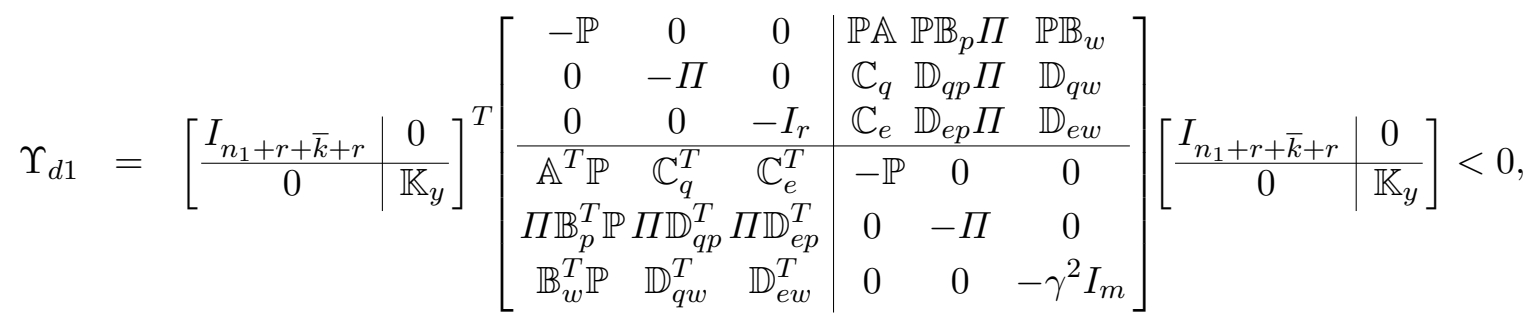

$$
\begin{aligned}
& \Upsilon_{d 2}=\left[\begin{array}{c|c}
\mathbb{K}_{u} & 0 \\
\hline 0 & I_{n_{1}+r+\bar{k}+m}
\end{array}\right]^{T}\left[\begin{array}{ccc|ccc}
-\mathbb{Q} & 0 & 0 & \mathbb{A} \mathbb{B}_{p} \Pi^{-1} & \mathbb{B}_{w} \\
0 & -\Pi^{-1} & 0 & \mathbb{C}_{q} \mathbb{Q} \mathbb{D}_{q p} \Pi^{-1} & \mathbb{D}_{q w} \\
0 & 0 & -I_{r} & \mathbb{C}_{e} \mathbb{Q}_{e p} \Pi^{-1} & \mathbb{D}_{e w} \\
\hline \mathbb{Q A}^{T} & \mathbb{Q C}_{q}^{T} & \mathbb{Q C}_{e}^{T} & -\mathbb{Q} & 0 & 0 \\
\Pi^{-1} \mathbb{B}_{p}^{T} \Pi^{-1} \mathbb{D}_{q p}^{T} \Pi^{-1} \mathbb{D}_{e p}^{T} & 0 & -\Pi^{-1} & 0 \\
\mathbb{B}_{w}^{T} & \mathbb{D}_{q w}^{T} & \mathbb{D}_{e w}^{T} & 0 & 0 & -\gamma^{2} I_{m}
\end{array}\right] \\
& \times\left[\begin{array}{c|c}
\mathbb{K}_{u} & 0 \\
\hline 0 & I_{n_{1}+r+\bar{k}+m}
\end{array}\right]<0,
\end{aligned}
$$

where $\mathbb{K}_{y}$ and $\mathbb{K}_{u}$ are two matrices whose columns span the null spaces of $\left[\begin{array}{lll}\mathbb{C}_{y} & \mathbb{D}_{y p} & \mathbb{D}_{y w}\end{array}\right]$ and $\left[\begin{array}{lll}\mathbb{B}_{u}^{T} & 0 & 0\end{array}\right]$, respectively. All gains $Z$ are given by (58) where matrices $\mathbb{K}, \mathbb{S}_{1}, \mathbb{S}_{2}$ are given by (59a), (59b) and (59c). Matrices $\overline{\mathbb{B}}, \overline{\mathbb{C}}$ and $\overline{\mathbb{Q}}$ are given by

$$
\left[\begin{array}{cc}
\mathbb{B} & \overline{\mathbb{Q}} \\
\bullet & \overline{\mathbb{C}}
\end{array}\right]=\left[\begin{array}{c|cccccc}
-\mathbb{B}_{u} & -\mathbb{Q} & 0 & 0 & \mathbb{A} \mathbb{Q} & \mathbb{B}_{p} \Pi^{-1} & \mathbb{B}_{w} \\
0 & 0 & -\Pi^{-1} & 0 & \mathbb{C}_{q} \mathbb{Q} \mathbb{D}_{q p} \Pi^{-1} & \mathbb{D}_{q w} \\
0 & 0 & 0 & -I_{r} & \mathbb{C}_{e} \mathbb{Q}^{-} \mathbb{D}_{e p} \Pi^{-1} & \mathbb{D}_{e w} \\
0 & \mathbb{Q A}^{T} & \mathbb{Q C}_{q}^{T} & \mathbb{Q C}_{e}^{T} & -\mathbb{Q} & 0 & 0 \\
0 & \Pi^{-1} \mathbb{B}_{p}^{T} \Pi^{-1} \mathbb{D}_{q p}^{T} \Pi^{-1} \mathbb{D}_{e p}^{T} & 0 & -\Pi^{-1} & 0 \\
0 & \mathbb{B}_{w}^{T} & \mathbb{D}_{q w}^{T} & \mathbb{D}_{e w}^{T} & 0 & 0 & -\gamma^{2} I_{m} \\
\hline \bullet & 0 & 0 & 0 & \mathbb{C}_{y} \mathbb{Q} \mathbb{D}_{y p} \Pi^{-1} & \mathbb{D}_{y w}
\end{array}\right] .
$$

$\mathbb{R}_{1}, \mathbb{R}_{2}$ and $\mathbb{Z}$ are arbitrary matrices of appropriate dimensions satisfying $\mathbb{R}_{1}=\mathbb{R}_{1}^{T}>0$ and $\left\|\mathbb{R}_{2}\right\|<1$. Matrices $\overline{\mathbb{B}}_{L}, \overline{\mathbb{B}}_{R}, \overline{\mathbb{C}}_{L}$ and $\overline{\mathbb{C}}_{R}$ are any full rank matrices such that $\overline{\mathbb{B}}=\overline{\mathbb{B}}_{L} \overline{\mathbb{B}}_{R}$ and $\overline{\mathbb{C}}=\overline{\mathbb{C}}_{L} \overline{\mathbb{C}}_{R}$.

Proof. The proof is similar to that of theorem 2.

Remark 2. Notice that the constraints (57c) and (62c) are not convex. The relation is a bilinearity but it is of different form from that of the filter derivation (see section 2.3, equation (31)). In section 2.3 we have intrinsic bilinearity (in fact it is a nonlinearity of "type $Z^{2}$ ") without any well-known tool for solving it whereas in the case of the static output feedback (see equations (57c) and (62c)), there exists some heuristic which can be applied to try to solve the bilinearity. Thus in section 4 , the matrices $\mathbb{P}$ and $\mathbb{Q}$ are obtained by using the cone complementary linearization technique El Ghaoui et al. (1997).

\section{Numerical example}

In this section, we give a numerical example in the continuous-time case to illustrate our approach for the synthesis of an unbiased robust reduced order $\mathcal{H}_{\infty}$ filter for a linear system having uncertain variables described by IQC. 
Let us consider the following uncertain linear system (see (1))

$$
\begin{aligned}
& \dot{x}_{1}=\left[\begin{array}{ccc}
-10 & 1 & 0 \\
2 & -12 & 1 \\
0 & -3 & -2
\end{array}\right] x_{1}+\left[\begin{array}{c}
0.7 \\
-0.1 \\
-0.5
\end{array}\right] p_{1}+\left[\begin{array}{c}
0.7 \\
-0.1 \\
-0.5
\end{array}\right] p_{2}+\left[\begin{array}{c}
0.7 \\
-0.1 \\
-0.5
\end{array}\right] p_{3}+\left[\begin{array}{ccc}
0 & 1 & 0 \\
0 & 0 & 0 \\
4 & -1 & 1
\end{array}\right] w \\
& \dot{x}_{2}=\left[\begin{array}{lll}
-4 & 0 & -5
\end{array}\right] x_{1}+0.5 x_{2}+0.7 p_{1}+0.8 p_{2}+0.3 p_{3}+\left[\begin{array}{ccc}
2 & 1 & 0
\end{array}\right] w \\
& y=\left[\begin{array}{ccc}
-2 & 0 & 1 \\
1 & 1 & 0
\end{array}\right] x_{1}+\left[\begin{array}{l}
0 \\
1
\end{array}\right] x_{2}+\left[\begin{array}{c}
0.1 \\
-0.1
\end{array}\right] p_{1}+\left[\begin{array}{c}
0.1 \\
-0.1
\end{array}\right] p_{2}+\left[\begin{array}{c}
0.1 \\
-0.1
\end{array}\right] p_{3}+\left[\begin{array}{ccc}
1 & 0 & 0 \\
-1 & 0 & 0
\end{array}\right] w \\
& z=\left[\begin{array}{ccc}
0 & 1 & -10 \\
1 & -1 & 2
\end{array}\right] x_{1}+\left[\begin{array}{c}
1 \\
0.5
\end{array}\right] x_{2}
\end{aligned}
$$

with the uncertainties $p_{i}(t) \in \mathbb{R}, i=1, \ldots, 3$ (that is $k_{1}=k_{2}=k_{3}=1, \bar{k}=k_{1}+k_{2}+k_{3}=3$ ) and $p_{1}(t) \neq p_{2}(t) \neq p_{3}(t) . A_{2}=0.5$ is unstable (see item (ii) of assumption 1 ).

To describe the effect of these uncertainties on the system (64), we take three unknown functions of time $f_{1}(t), f_{2}(t)$ and $f_{3}(t)$ belonging to $[-1,1], \forall t \geqslant 0$. In the nominal case, $f_{i}(t)=0$. Notice that $q_{i}(t)=p_{i}(t) / f_{i}(t)\left(f_{i}(t) \neq 0\right.$ except for the nominal case $)$ are fictitious outputs given by

$$
q_{i}=E_{11 i} x_{1}+E_{2 i} p+E_{3 i} w \quad i=1, \ldots, 3 .
$$

So $p_{1}(t), p_{2}(t)$ and $p_{3}(t)$ are as follows

$$
p_{i}=f_{i}\left(E_{11 i} x_{1}+E_{2 i} p+E_{3 i} w\right) \quad i=1, \ldots, 3
$$

and satisfy the admissible IQC given by (6) for $i=1, \ldots, 3$ where (see (4))

$$
\left[\begin{array}{lll}
E_{111} & E_{21} & E_{31} \\
E_{112} & E_{22} & E_{32} \\
E_{113} & E_{23} & E_{33}
\end{array}\right]=\left[\begin{array}{ccc:ccc:ccc}
1 & 0.2 & 0.1 & 0.02 & 0.03 & 0.01 & 0.3 & 0.1 & 1 \\
\hdashline 0.1 & 0.3 & 0.1 & 0.02 & 0.05 & 0.03 & 0.2 & 0.1 & 0.2 \\
\hdashline 0.1 & 0.3 & 0.4 & 0.26 & 0.1 & 0.14 & 2 & 0.1 & 0.3
\end{array}\right] .
$$

The objective is to apply theorem 2 to obtain a robust functional unbiased $\mathcal{H}_{\infty}$ filter for the system (64).

First of all, it is obvious that condition (36) is verified. Relations (57a), (57b) and (57c) are solved using the (iterative) cone complementarity method El Ghaoui et al. (1997). After three iterations, we get

$$
\mathbb{P Q}=\left[\begin{array}{ccccc}
1 & -1.244 \times 10^{-11} & -1.278 \times 10^{-11} & 5.606 \times 10^{-11} & 5.777 \times 10^{-11} \\
6.861 \times 10^{-12} & 1 & 6.726 \times 10^{-11} & 6.642 \times 10^{-11} & -1.106 \times 10^{-10} \\
1.013 \times 10^{-11} & -1.010 \times 10^{-11} & 1 & 1.941 \times 10^{-10} & 6.051 \times 10^{-11} \\
-7.385 \times 10^{-13} & 5.176 \times 10^{-13} & 4.822 \times 10^{-13} & 1 & 1.738 \times 10^{-11} \\
1.781 \times 10^{-12} & -1.135 \times 10^{-12} & 3.390 \times 10^{-12} & -2.541 \times 10^{-11} & 1
\end{array}\right]
$$

where

$$
\begin{aligned}
\mathbb{P} & =\left[\begin{array}{ccccc}
5.2548118 & -10.986408 & 1.6067552 & -0.0473975 & -0.0806303 \\
-10.986408 & 77.091606 & -6.9391407 & 0.1738354 & -0.2137849 \\
1.6067552 & -6.9391407 & 2.5020225 & -0.0332746 & 0.1270482 \\
-0.0473975 & 0.1738354 & -0.0332746 & 0.0557124 & -0.1848252 \\
-0.0806303 & -0.2137849 & 0.1270482 & -0.1848252 & 0.8017079
\end{array}\right], \\
\mathbb{Q} & =\left[\begin{array}{ccccc}
0.3067791 & 0.0321291 & -0.1088573 & 1.2064261 & 0.3348008 \\
0.0321291 & 0.0209583 & 0.0373041 & -0.0260725 & -0.0031023 \\
-0.1088573 & 0.0373041 & 0.5765746 & -0.7274441 & -0.2600759 \\
1.2064261 & -0.0260725 & -0.7274441 & 82.421173 & 19.230982 \\
0.3348008 & -0.0031023 & -0.2600759 & 19.230982 & 5.7548939
\end{array}\right],
\end{aligned}
$$

and with $\mu_{1}=9.4, \mu_{2}=17.65, \mu_{3}=17.65$ and $\gamma=17.3$. 
Next, using relations (58), (59a), (59b), (59c) and (59d), we obtain the following gain matrix $Z$

$$
Z=\left[\begin{array}{llll}
13.106351 & 13.106351 & 29.957374 & 99.233803 \\
3.3102008 & 3.3102008 & 7.5661734 & 25.062949
\end{array}\right]
$$

It remains to use relations (33) and (35) to deduce the filter matrices (8) which are

$N=\left[\begin{array}{cc}-34.492857 & -142.51574 \\ -6.9317996 & -35.986032\end{array}\right], J=\left[\begin{array}{cc}-790.76245 & -717.93454 \\ -173.13327 & -161.7179\end{array}\right], E=\left[\begin{array}{cc}11.89537 & 11.89537 \\ 2.8623665 & 2.8623665\end{array}\right]$.

To illustrate the behaviour of the robust reduced order $\mathcal{H}_{\infty}$ filter defined by $(67), f(t)$ is chosen as follows

$$
f(t)=\left[\begin{array}{ccc}
f_{1}(t) & 0 & 0 \\
0 & f_{2}(t) & 0 \\
0 & 0 & f_{3}(t)
\end{array}\right]=\left[\begin{array}{ccc}
\alpha & 0 & 0 \\
0 & -\alpha & 0 \\
0 & 0 & \frac{2 \alpha}{2.1}
\end{array}\right]
$$

for constant values of $\alpha \in[-1,1]$. Then, the maximum singular values of the transfer between the reconstruction error $e(t)$ and the disturbance $w(t)$ are drawn in figure 1.

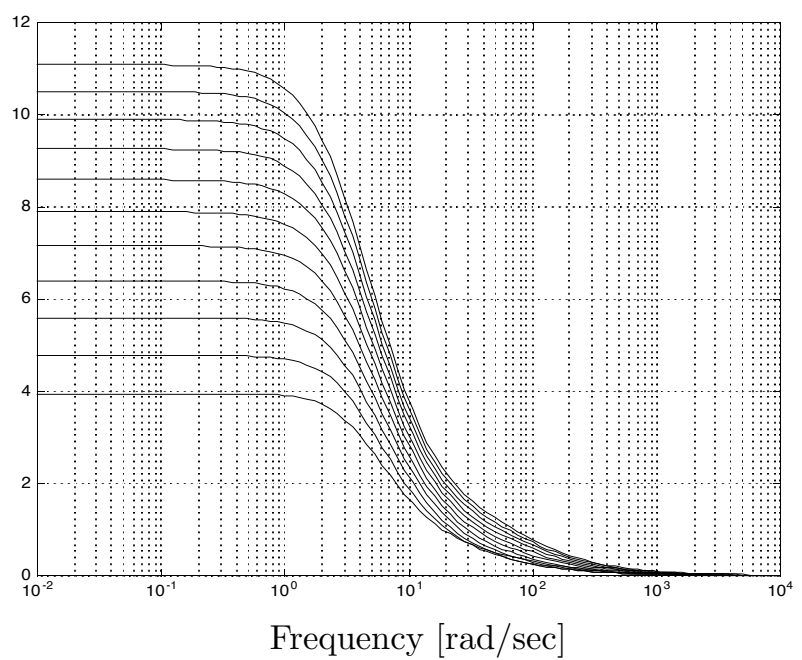

Figure 1: Maximum singular values of the transfer functions from $w(t)$ to $e(t)$ for $\alpha \in[-1,1]$ (with an increment of 0.2).

The maximal $\mathcal{L}_{2}$ gain of the filter defined by $(67)$ is given by $\gamma=11.0775$. This values is lower than $\gamma=17.3$ which was used during the filter synthesis. It is probably possible to solve the problem with $\gamma<17.3$ but there is some conservatism due to the use of the cone complementary heuristic El Ghaoui et al. (1997).

For the simulations purpose, we use $\alpha=-1$ in the uncertain case and $\alpha=0$ in the nominal one. The disturbance $w(t)$ is given by figure 2 . 


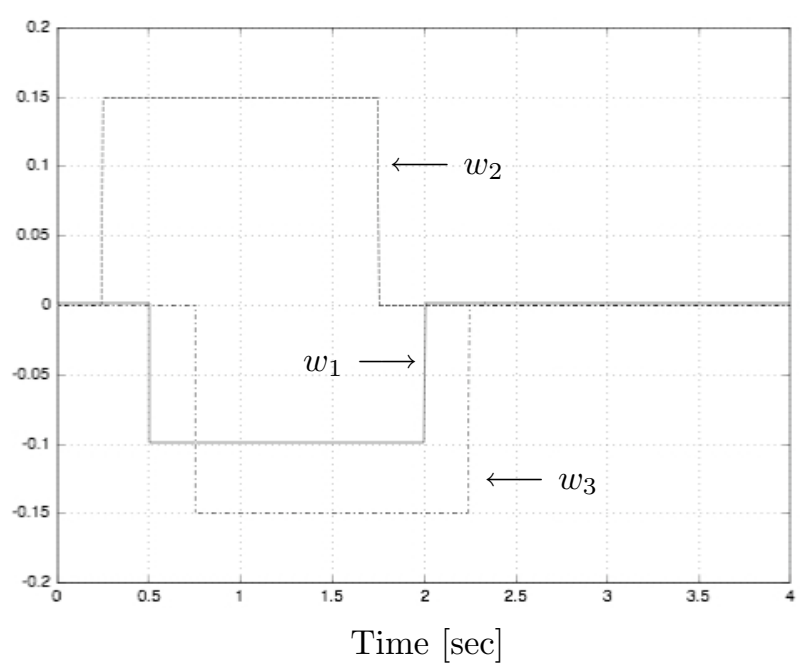

Figure 2: Unmeasurable perturbation $w(t)$.

For scaling reasons, the reconstruction error $e(t)$ obtained with the robust $\mathcal{H}_{\infty}$ filter is described by figures 3 and 4 for the uncertain system $(\Delta \neq 0)$ and the nominal one $(\Delta=0)$.

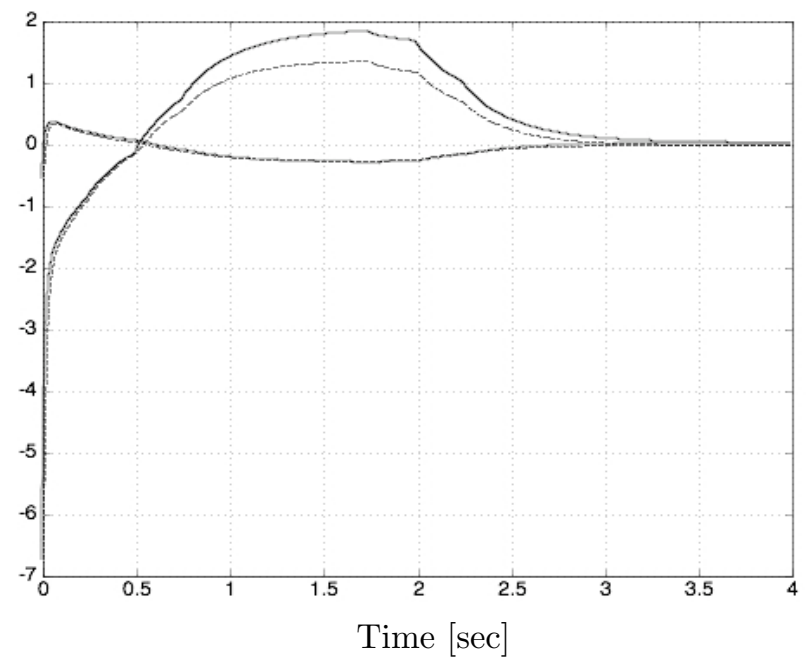

Figure 3: Reconstruction error $e(t)$ for the robust $\mathcal{H}_{\infty}$ filter : nominal and uncertain systems.

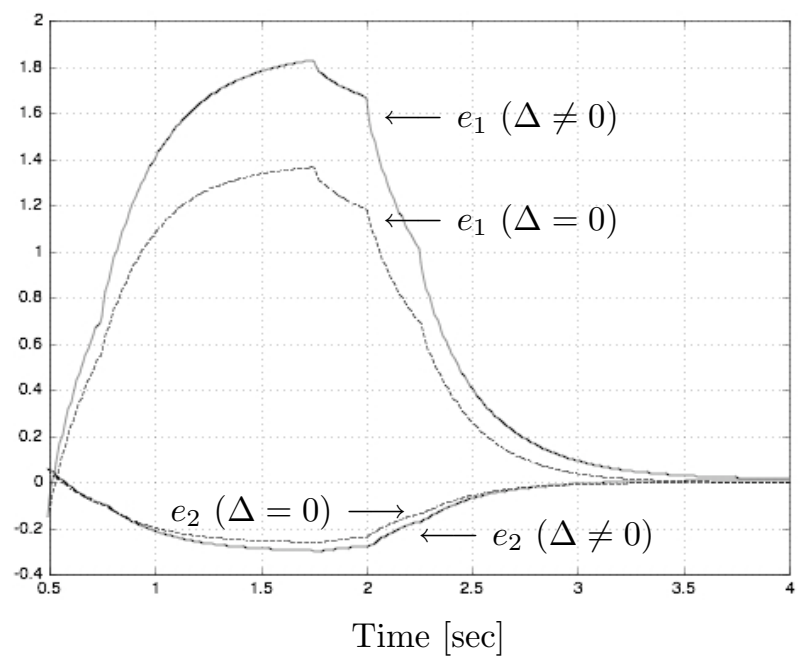

Figure 4: Zoom on the reconstruction error $e(t)$ given in figure 3. 


\section{Conclusion}

A method to design a robust unbiased $\mathcal{H}_{\infty}$ reduced order filter is given in this paper. The uncertainties of the system are described by Integral Quadratic Constraints (IQC). The filter is of the same order as the functional to be estimated. The unbiasedness of the proposed filter is obtained from the error dynamics and permits to parameterize all the filter matrices through a single gain matrix. An additional constraint on the filter structure is added to avoid bilinearities in the filtering error and the unbiased filter is shown to converge despite the presence of unstable mode in the system state. Then the robust filtering problem is transformed into a static output feedback one. So, LMI method is applied with the cone complementary linearization heuristic to obtain the filter matrices. A numerical example is given to show the effectiveness of the proposed approach.

\section{References}

S. Bittanti and F.A. Cuzzola, "Unbiased robust $\mathcal{H}_{\infty}$ filtering by means of LMI optimisation", in IFAC Symposium on Robust Control Design, Prague, Czech Republic, 2000.

P. Bolzern, P. Colinari and G. De Nicolao, "Optimal robust filtering with time-varying parameter uncertainty", International Journal of Control, 63, pp. 557-576, 1996.

M. Darouach, "Existence and design of functional observers for linear systems", IEEE Transactions on Automatic Control, 45, pp. 940-943, 2000.

M. Darouach, M. Zasadzinski and H. Souley Ali, "Robust reduced order unbiased filtering via LMI", in European Control Conference, Porto, Portugal, 2001.

C.E. de Souza, "H $\mathcal{H}_{\infty}$ filter design for linear time-delay system", in Analyse et Commande des Systèmes à Retard, Ecole d'Eté d'Automatique de Grenoble (LAG), Grenoble, France, 2000.

L. El Ghaoui, F. Oustry and M. Aït Rami, "A cone complementary linearization algorithm for static output-feedback and related problems", IEEE Transactions on Automatic Control, 42, pp. 1171-1176, 1997.

L. El Ghaoui and G. Calafiore, "Robust filtering for discrete-time systems with bounded noise and parametric uncertainties", IEEE Transactions on Automatic Control, 46, pp. 1084-1089, 2001.

J.C. Geromel, "Optimal linear filtering under parameter uncertainty", IEEE Transactions on Signal Processing, 47, pp. 168-175, 1999.

J.C. Geromel, J. Bernussou, G. Garcia and M.C. de Oliveira, " $\mathcal{H}_{2}$ and $\mathcal{H}_{\infty}$ robust filtering for discretetime linear systems", SIAM Journal on Control and Optimization, 38, pp. 1353-1368, 2000.

K.M. Grigoriadis and J.T. Watson, "Reduced-order $\mathcal{H}_{\infty}$ and $\mathcal{L}_{2}-\mathcal{L}_{\infty}$ filtering via linear matrix inequalities", IEEE Transactions on Aerospace and Electronic Systems, 33, pp. 1326-1338, 1997.

T. Iwasaki and R.E. Skelton, "All controllers for the general $\mathcal{H}_{\infty}$ control problems : LMI existence conditions and state space formulas", Automatica, 30, pp. 1307-1317, 1994.

H. Kim, C.S. Sims and K.M. Nagpal, "Reduced order filtering in an $\mathcal{H}_{\infty}$ setting", in IEEE American Control Conference, Chicago, USA, 1992.

P. Lancaster and M. Tismenetsky, The Theory of Matrices, 2nd edition, Academic Press, Orlando, USA, 1985.

H. Li and M. Fu, "A linear matrix inequality approach to robust $\mathcal{H}_{\infty}$ filtering", IEEE Transactions on Signal Processing, 45, pp. 2338-2350, 1997. 
K.M. Nagpal and P.P. Khargonekar, "Filtering and smoothing in an $\mathcal{H}_{\infty}$ setting", IEEE Transactions on Automatic Control, 36, pp. 152-166, 1991.

K.M. Nagpal, R.E. Helmick and C.S. Sims, "Reduced-order estimation, Part I : filtering", International Journal of Control, 45, pp. 1867-1888, 1987.

J. O'Reilly, Observers for Linear Systems, Academic Press, New York, 1983.

A.V. Savkin and I.R. Petersen, "Robust state estimation for uncertain systems with averaged integral quadratic constraints", International Journal of Control, 64, pp. 923-940, 1996.

A.V. Savkin and I.R. Petersen, "Fixed-order robust filtering for linear uncertain systems", Automatica, 33, pp. 253-254, 1997.

U. Shaked, "H $\mathcal{H}_{\infty}$-minimum error state estimation of linear stationary processes", IEEE Transactions on Automatic Control, 35, pp. 554-558, 1990.

U. Shaked and C.E. de Souza, "Robust minimum variance filtering", IEEE Transactions on Signal Processing, 43, pp. 2474-2483, 1995.

U. Shaked and Y. Theodor, "A frequency domain approach to the problem of $\mathcal{H}_{\infty}$-minimum error state estimation and deconvolution", IEEE Transactions on Signal Processing, 40, pp. 3001-3011, 1992.

H.D. Tuan, P. Apkarian and T.Q. Nguyen, "Robust and reduced order filtering : new LMI-based characterizations and methods", IEEE Transactions on Signal Processing, 49, pp. 2975-2984, 2001.

F. Wang and V. Balakrishnan, "Robust Kalman filters for linear time-varying systems with stochastic parametric uncertainties", IEEE Transactions on Signal Processing, 50, pp. 803-813, 2002.

J.T. Watson and K.M. Grigoriadis, "Optimal unbiased filtering via linear matrix inequalities", Systems and Control Letters, 35, pp. 111-118, 1998.

L. Xie, "Output feedback $\mathcal{H}_{\infty}$ control of systems with parameter uncertainty", International Journal of Control, 63, pp. 741-750, 1996.

L. Xie, C.E. de Souza and Y.C. Soh, "Robust filtering for uncertain systems with unstable modes", in IEEE Conference on Decision and Control, Lake Buena Vista, USA, 1994.

L. Xie, M. Fu and H. Li, "Passivity analysis and passification for uncertain processing systems", IEEE Transactions on Signal Processing, 46, pp. 2394-2403, 1998. 